\title{
Dermatitis herpetiforme: manifestación específica de la enfermedad celiaca
}

\section{Dermatitis herpetiformis: specific manifestation of celiac disease}

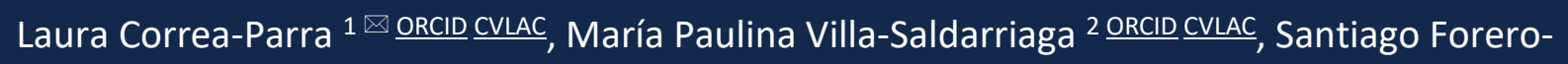
Saldarriaga ${ }^{3}$ ORCID CVLAC

${ }^{1}$ Medicina General, Coomsocial IPS, Medellín, Colombia.

${ }^{2}$ Medicina General, Home Group SAS, Medellín, Colombia.

${ }^{3}$ Residente Medicina Interna, Fundación Universitaria Sanitas, Clínica Universitaria Colombia, Bogotá, Colombia;

Grupo de enfermedades infecciosas y crónicas (GEINCRO), Fundación Universitaria San Martín.

Fecha correspondencia:

Recibido: agosto 04 de 2021.

Revisado: septiembre 08 de 2021.

Aceptado: septiembre 14 de 2021.

Forma de citar:

Correa-Parra L, Villa-Saldarriaga

MP, Forero-Saldarriaga S.

Dermatitis herpetiforme:

manifestación específica de la

enfermedad celiaca. Rev CES Med.

2021; 35(3). 272-283.

https://dx.doi.org/10.21615/

cesmedicina.6352

Open access

C Derecho de autor

Licencia creative commons

Ética de publicaciones

Revisión por pares

Gestión por Open Journal System DOI: $10.21615 /$ cesmedicina.6352

ISSNe 2215-9177

ISSN 0120-8705

Publica con nosotros

\section{Resumen}

La enfermedad celíaca es un trastorno sistémico con base autoinmune, secundario a la exposición continua al gluten en personas con susceptibilidad genética, que lleva a desarrollo de anticuerpos específicos, afectando principalmente el intestino delgado con la subsecuente enteropatía. Las manifestaciones clínicas incluyen síntomas digestivos y manifestaciones extra intestinales. En este último grupo se destacan las manifestaciones dermatológicas, específicamente la dermatitis herpetiforme, la cual es considerada específica de la enfermedad celíaca. Clínicamente, se presenta como un exantema papulovesicular pruriginoso. El diagnóstico se basa en la biopsia de piel con inmunofluorescencia directa y la presencia de anticuerpos anti-transglutaminasa tisular 2 y solo en casos específicos se debe complementar con otros estudios. El tratamiento se basa en dieta libre de gluten, que mejora la sintomatología, controla y previene las complicaciones de la enfermedad celíaca, como las neoplasias digestivas y el desarrollo de otras enfermedades autoinmunes.

Palabras clave: enfermedad celíaca; enfermedades de la piel; dermatitis herpetiforme; dieta sin gluten. 


\section{Abstract}

Celiac disease is a systemic disorder with an autoimmune basis, secondary to continuous exposure to gluten in people with genetic susceptibility, which leads to the development of specific antibodies mainly affecting the small intestine with subsequent enteropathy. The clinical manifestations include digestive symptoms and extra intestinal manifestations. In this last group, the dermatological manifestations stand out, specifically dermatitis herpetiformis, which is considered specific to Celiac disease. It presents clinically as a pruritic papulovesicular rash. The diagnosis is based on a skin biopsy with direct immunofluorescence and the presence of anti-tissue transglutaminase 2 antibodies, only in specific cases it should be complemented with other studies. Treatment is based on a gluten-free diet, which improves symptoms, controls and prevents complications of Celiac disease, such as digestive neoplasms and the development of other autoimmune diseases.

Keywords: celiac disease; skin diseases; dermatitis herpetiformis; diet; gluten-free.

\section{Introducción}

La enfermedad celíaca es un trastorno sistémico con base autoinmune secundaria a la exposición continua al gluten obtenido de la dieta en personas con susceptibilidad genética, en las cuales hay desarrollo de anticuerpos específicos, afectando principalmente el intestino delgado con la subsecuente enteropatía (1). La enfermedad celíaca ha sido reconocida desde hace más de 100 años; sin embargo, fue en la década de 1950 cuando el pediatra holandés Willem Karel Dicke estableció un vínculo entre el componente proteico de la exposición al gluten y la enfermedad celíaca ${ }^{(2)}$. Antes se consideraba una enfermedad rara que afectaba principalmente a los niños, y actualmente se reconoce como uno de los trastornos auto inmunitarios más comunes, estimándose que afecta cerca del $1 \%$ de la población mundial (3); en Latinoamérica, específicamente en nuestro medio, se reporta una prevalencia del $0,46 \%$ a $0,64 \%{ }^{(4)}$.

Los síntomas digestivos son la presentación clínica clásica de la enfermedad celíaca ${ }^{(5)}$. Paradójicamente, es la que se presenta con menor frecuencia, por lo que las manifestaciones extra intestinales -consideradas la presentación clínica no clásica- toman gran importancia, ya que su reconocimiento podría ayudar a un diagnóstico temprano y oportuno y reducir así el riesgo de neoplasias digestivas, que son más frecuentes en pacientes con enfermedad celíaca con respecto a la población general ${ }^{(6)}$. En esta revisión de tema se abordará la presentación dermatológica de la enfermedad celíaca, específicamente la dermatitis herpetiforme. 


\section{Patogenia}

La patogenia se basa en una respuesta autoinmune desencadenada por el gluten de la dieta en personas con predisposición genética ${ }^{(3)}$. Aproximadamente, el $95 \%$ de los pacientes con enfermedad celíaca expresan antígenos leucocitarios humanos (por sus siglas en inglés, HLA), IDQ2 en un 90 - 95 \% y DQ8 en un 5-10\%; sin embargo, su presencia se considera necesaria pero no suficiente para desarrollar la enfermedad, ya que la mayoría de personas HLA-DQ2 o DQ8 positivos no presentan la enfermedad ${ }^{(3,7)}$.

El gluten es un conjunto de proteínas presentes en algunos cereales, el cual es digerido de manera incompleta por las peptidasas gástricas, pancreáticas y del borde en cepillo dejando como resultado péptidos ${ }^{(8)}$. Estos atraviesan la pared intestinal y son deaminados por la transglutaminasa tisular generando fragmentos de gliadina. Posteriormente, estos fragmentos son presentados y unidos a células presentadoras de antígenos que expresan las moléculas HLA- DQ2 o DQ8, lo que lleva a la activación de los linfocitos T y a la producción de citoquinas que generan daño tisular a nivel intestinal, resultando en atrofia de las vellosidades, inflamación de la mucosa y disminución en la absorción de nutrientes ${ }^{(1)}$. También se produce activación de linfocitos $B$, los cuales generan autoanticuerpos contra la transglutaminasa conocidos como anticuerpos anti-transglutaminasa tisular 2 (anti-TG2 o tTG) y otros anticuerpos contra la gliadina y el endomisio conocidos como anticuerpos anti-péptidos de gliadina deaminada (anti-DGP) y anticuerpos anti-endomisio (anti-EMA), respectivamente ${ }^{(1)}$.

En la dermatitis herpetiforme el principal autoantígeno es la transglutaminasa epidérmica (eTG), enzima que se expresa fisiológicamente en la capa espinosa de la epidermis y contribuye a la diferenciación epidérmica terminal ${ }^{(9)}$; sin embargo, la especificidad de los anticuerpos contra la eTG, para el diagnóstico de dermatitis herpetiforme aún no se ha confirmado por lo que esta prueba de anticuerpos no se utiliza en la práctica clínica (10-11). Aún persisten controversias sobre los mecanismos por los cuales se desarrollan los autoanticuerpos anti-eTG. Se cree que ocurre en el intestino, probablemente como resultado de un fenómeno de propagación del epítopo, debido a la alta homología de secuencia entre transglutaminasas tisulares $^{(9)}$.

\section{Presentación clínica}

La enfermedad celíaca se clasifica según los criterios de Oslo, los cuales recomiendan el uso de "enfermedad celíaca clásica" y "no clásica" (12). Los síntomas clásicos de la enfermedad celíaca incluyen diarrea crónica, esteatorrea, dolor abdominal, pérdida de peso y falla en el medro. Los síntomas no clásicos se presentan con mayor frecuencia y se dividen en gastrointestinales y no 
gastrointestinales. En el primer grupo se encuentran síntomas dispépticos, reflujo gastroesofágico, síntomas similares al síndrome de colon irritable, entre otros. Entre los no gastrointestinales se incluyen el compromiso de diferentes sistemas como neurológico, cardiopulmonar, renal, endocrinológico, dermatológico, etc. ${ }^{(8,13)}$ La anemia por deficiencia de hierro es el signo extra intestinal más común en adultos con enfermedad celíaca y se presenta en el $48 \%$ de los pacientes ${ }^{(14)}$ (tabla 1 ).

Tabla 1. Presentación no clásica de la enfermedad celíaca.

\begin{tabular}{ll}
\hline Sistema & Manifestación \\
\hline Hematológica & $\begin{array}{l}\text { Anemia ferropénica, anemia megaloblástica por deficiencia de vitamina } \\
\text { b12 y folatos }\end{array}$ \\
\hline Neurológica & $\begin{array}{l}\text { Ataxia cerebelosa, neuropatía periférica, epilepsia, deterioro cognitivo, } \\
\text { demencia, migraña, Ansiedad, depresión, déficit de atención e } \\
\text { hiperactividad, fatiga crónica }\end{array}$ \\
\hline Dermatológica & $\begin{array}{l}\text { Dermatitis herpetiforme, pustulosis palmo plantar, estomatitis aftosa, } \\
\text { psoriasis, dermatitis atópica, vitiligo, vasculitis de pequeños vasos, } \\
\text { urticaria idiopática y alopecia areata }\end{array}$ \\
\hline Osteomusculares & osteoporosis, mialgias, artralgias y artritis \\
\hline Cardiopulmonar & $\begin{array}{l}\text { Derrame pericárdico, miocarditis y miocardiopatía, Hemosiderosis } \\
\text { pulmonar }\end{array}$ \\
\hline Renal & Nefropatía por lgA \\
\hline Endocrinológico & Enfermedades de la tiroides, enfermedad de Addison, diabetes tipo 1 \\
\hline Reproductivo & $\begin{array}{l}\text { Amenorrea, menarquia tardía, pubertad tardía, menopausia precoz, } \\
\text { esterilidad }\end{array}$ \\
\hline Hepatitis celíaca, esteatosis
\end{tabular}

Adaptado de Therrien A. ${ }^{(5)}$ y Muddasani S. (15) 


\section{Dermatitis herpetiforme}

La dermatitis herpetiforme es la manifestación dermatológica más frecuente de la enfermedad celíaca, con una prevalencia que puede llegar hasta el $13 \%{ }^{(10,16)}$. Es una enfermedad ampollosa autoinmune, descrita por Louis Dühring en $1884^{(9)}$. Se presenta predominantemente en personas caucásicas y es muy rara entre las poblaciones africanas y asiáticas ${ }^{(9)}$. La mayor incidencia se da entre hombres entre 60 y 69 años y mujeres entre 50 y 59 años ${ }^{(5)}$.

La mayoría de pacientes con dermatitis herpetiforme no presentan sintomatología digestiva, pero hasta un $70 \%$ presentan enteropatía con alteraciones histopatológicas en la biopsia intestinal $^{(11,17)}$. Los pacientes con dermatitis herpetiforme también tienen un mayor riesgo de linfomas no Hodgkin y neoplasias malignas gastrointestinales ${ }^{(9)}$.

La dermatitis herpetiforme se manifiesta como una erupción polimórfica papulovesicular, eritematosa, intensamente pruriginosa, con distribución herpetiforme y simétrica, en superficies extensoras de las extremidades, glúteos y región sacra; también puede comprometer el cuello, cuero cabelludo, abdomen, parte superior de la espalda y hombros ${ }^{(9)}$. Las vesículas pueden fusionarse en pequeñas ampollas tensas con contenido sero-hemorrágico ${ }^{(9)}$. Se pueden producir excoriaciones, erosiones y costras como lesiones secundarias al rascado y ruptura de vesículas o ampollas; estas últimas, generalmente curan sin dejar cicatriz, pero en algunas ocasiones pueden dejar hipo e hiperpigmentación postinflamatoria ${ }^{(9)}$. El compromiso oral es raro, se manifiesta principalmente en erosiones que afectan tanto a la mucosa oral como a la lengua, asociado a dolor y ardor; no obstante, dichas manifestaciones orales pueden estar presentes en la enfermedad celíaca sin compromiso cutáneo ${ }^{(5,9)}$.

\section{Diagnóstico}

El diagnóstico de la enfermedad celíaca se basa en las pruebas serológicas y en la biopsia duodenal. La medición anticuerpos anti-transglutaminasa tisular IgA (IgA-tTG) tiene una alta sensibilidad (98\%) y un buen valor predictivo negativo ( $99 \%$ ), lo que lo hace el examen de primera línea para diagnóstico, por lo que un resultado negativo en pacientes con bajo riesgo de enfermedad celíaca descarta la enfermedad y evitaría la necesidad de realización de biopsia de intestino delgado ${ }^{(18)}$. Los anti-EMA y anti-DGP no se recomiendan de manera sistemática por su poca disponibilidad y alto costo ${ }^{(18)}$. 
La genotipificación de HLA puede ser útil en pacientes con resultados ambiguos, en pacientes con dieta libre de gluten o los que no se les puede realizar biopsia duodenal, y para evaluar familiares de primer grado de pacientes con enfermedad celíaca, debido a que la ausencia de HLA-DQ2 y HLA-DQ8 tiene un valor predictivo negativo del $98 \%(8,19,20)$.

La Sociedad Británica de Gastroenterología y las guías NICE recomiendan realizar pruebas serológicas a pacientes de alto riesgo, tales como pacientes con síntomas gastrointestinales inexplicados, pacientes con falla del crecimiento, fatiga prolongada, pérdida de peso inexplicada, úlceras en cavidad oral persistentes o severas, anemia inexplicada, diabetes mellitus tipo 1, enfermedad tiroidea autoinmune, síndrome de intestino irritable o familiares de primer grado con enfermedad celíaca ${ }^{(21-22)}$.

La endoscopia digestiva alta con biopsia duodenal sirve para establecer el diagnóstico definitivo solo en casos de duda diagnóstica en pacientes con alta sospecha (18). Los hallazgos histopatológicos sugestivos descritos son linfocitosis epitelial, hiperplasia de las criptas y atrofia vellosa ${ }^{(8)}$.

El diagnóstico de la dermatitis herpetiforme se basa en la biopsia de piel e inmunofluorescencia directa (IFD) ${ }^{(23)}$. Los hallazgos histológicos de la biopsia de piel en un tercio de los casos pueden ser inespecíficos, por lo que se considera la inmunofluorescencia directa el estándar de oro para el diagnóstico. Histológicamente, se presentan ampollas en vías de reepitelización, con micro abscesos e infiltrados neutrófilos en las papilas dérmicas. La inmunofluorescencia directa evidencia depósitos granulares de IgA en el vértice de las papilas y a lo largo de la membrana basal en la unión dermoepidérmica, considerándose un hallazgo patognomónico ${ }^{(9,11)}$. Los depósitos de IgG y C3 son menos característicos (23). Adicionalmente, el diagnóstico debe ir acompañado con anti-tTG y en casos específicos se necesitará otras ayudas diagnósticas que se muestran en la (figura 1) ${ }^{(23)}$. 


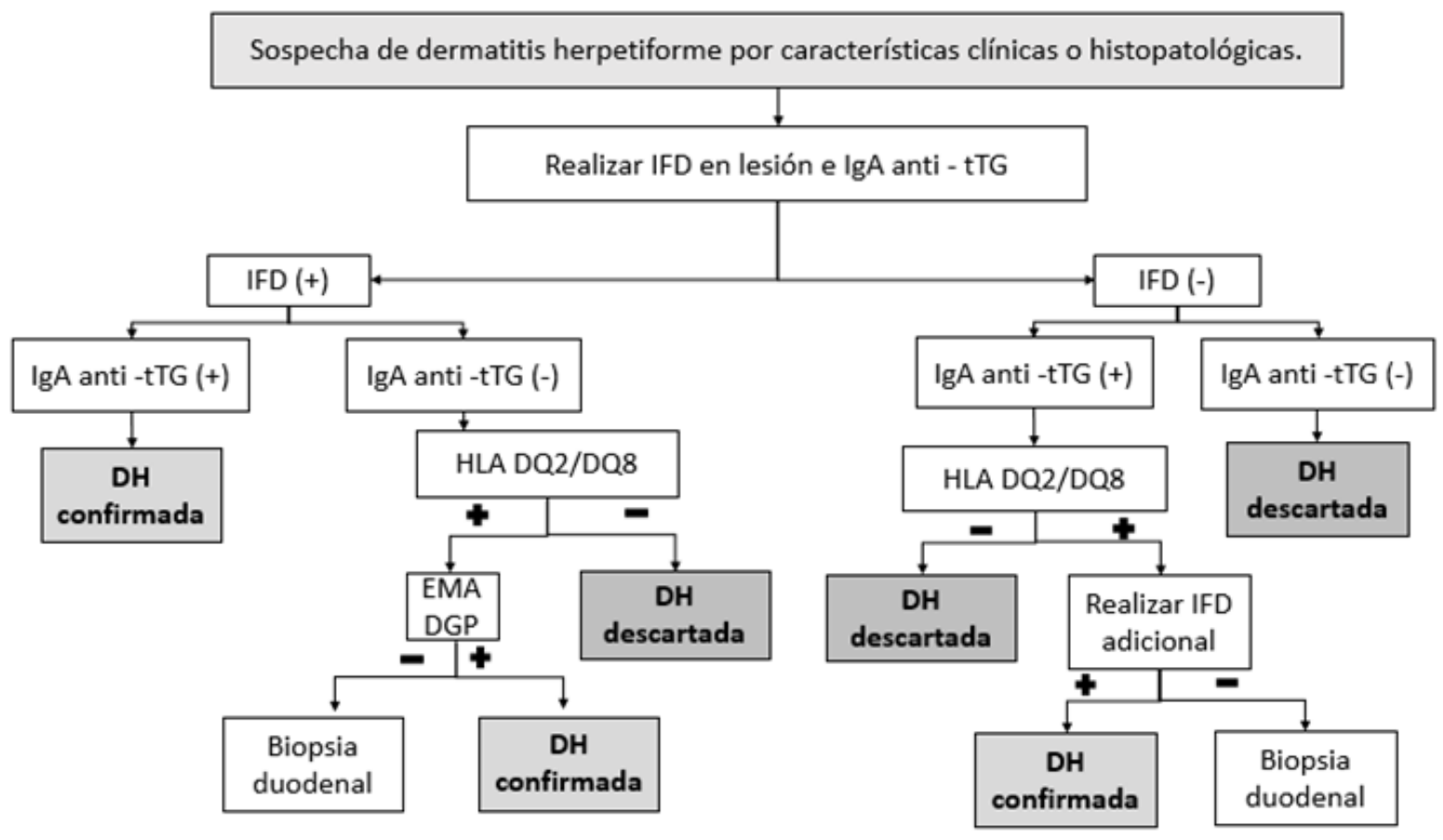

Figura 1. Algoritmo diagnóstico de la dermatitis herpetiforme.

DH: Dermatitis herpetiforme. DGP: anticuerpos anti-péptidos de gliadina deaminada. EMA: anticuerpos anti-endomisio. HLA: antígeno leucocitario humano. IFD: inmunofluorescencia directa. IgA anti tTG: inmunoglobulina A anti-transglutaminasa tisular. Adaptado de Antiga E. (23).

\section{Otras manifestaciones cutáneas}

Cada vez se notifican más enfermedades de la piel secundarias a la ingestión de gluten ${ }^{(9)}$. En general, las enfermedades dermatológicas comunes como psoriasis, dermatitis atópica, urticaria, estomatitis aftosa y rosácea se diagnostican con más frecuencia en los pacientes celíacos que en la población general; sin embargo, no es aconsejable realizar pruebas de cribado para enfermedad celíaca, ya que el riesgo relativo es bajo ${ }^{(24)}$.

Al tener un aspecto vesicular y al ser pruriginosa, la dermatitis herpetiforme puede confundirse con otras enfermedades más frecuentes; además, los traumas o escoriaciones por el rascado pueden distorsionar la semiología de la lesión lo que puede dificultar aún más el diagnóstico, por lo que es importante considerar diagnósticos diferenciales ${ }^{(25)}$ (tabla 2). 
Tabla 2. Diagnósticos diferenciales de la dermatitis herpetiforme.

\begin{tabular}{|c|c|}
\hline Enfermedad & Características \\
\hline $\begin{array}{l}\text { Eccema dishidrótico o de } \\
\text { Pomfólix }\end{array}$ & $\begin{array}{l}\text { Vesículas o bullas de inicio abrupto en palmas o plantas que } \\
\text { resuelven espontáneamente a las } 2 \text { o } 3 \text { semanas }\end{array}$ \\
\hline Escabiosis & Presencia de túneles y prurito predominantemente nocturno \\
\hline Herpes zoster & $\begin{array}{l}\text { Erupción vesicular unilateral en localización de dermatoma, } \\
\text { acompañado de dolor }\end{array}$ \\
\hline Herpes simple & $\begin{array}{l}\text { Flictenas o vesículas a veces dolorosas de localización } \\
\text { predominante en parte baja de la espalda, glúteos y espalda, } \\
\text { asociación con VIH }\end{array}$ \\
\hline Impétigo ampolloso & $\begin{array}{l}\text { Causado por } S \text {. Aureus coagulasa positivo, vesículas en cara } \\
\text { (alrededor de la boca y nariz) o en el lugar del trauma que } \\
\text { rompen con facilidad con costras mielicéricas }\end{array}$ \\
\hline Eritema multiforme & $\begin{array}{l}\text { Asociado a Virus de Herpes Simple, infecciones o drogas, } \\
\text { lesiones en diana en palmas, cuello y cara, en el } 70 \% \text { de los } \\
\text { casos afectan mucosas }\end{array}$ \\
\hline Lupus ampolloso & $\begin{array}{l}\text { Erupción de rápido inicio vesiculobullosa generalizada, } \\
\text { generalmente en tronco superior, extremidades superiores, } \\
\text { cuello y cara, prurito variable, asociación con síntomas } \\
\text { sistémicos }\end{array}$ \\
\hline Penfigoide ampolloso & $\begin{array}{l}\text { Mayor prevalencia en ancianos con enfermedades } \\
\text { neurológicas o psoriasis, se caracteriza por ampollas grandes y } \\
\text { tensas, difíciles de romper con signo de Nikolsky negativo, } \\
\text { sobre una base eritematosa acompañada de prurito, } \\
\text { predominio en abdomen, muslos y superficies de flexión de } \\
\text { las extremidades }\end{array}$ \\
\hline Dermatosis lineal por IgA & $\begin{array}{l}\text { Prurito prodrómico transitorio, vesículas o flictenas claras o } \\
\text { hemorrágicas en piel normal, eritematosa o con urticaria, } \\
\text { típicamente dispuestos en patrón herpetiforme o en "grupos } \\
\text { de joyas", asociado a síntomas oculares como ardor, secreción } \\
\text { o sensación de cuerpo extraño }\end{array}$ \\
\hline
\end{tabular}

Adaptado de Baptista A. (25) 


\section{Tratamiento}

El tratamiento de la enfermedad celíaca se basa en una dieta libre de gluten que debe estar guiada por personal experto en nutrición. La mejoría de los síntomas intestinales típicamente ocurre en días o semanas, mientras que la normalización de los marcadores serológicos al año de la dieta libre de gluten y la normalización histológica de la atrofia duodenal se presenta posteriormente ${ }^{(8)}$. Sin embargo, la mejoría clínica de la dermatitis herpetiforme puede tardar hasta uno a dos años y los síntomas pueden reaparecer con solo 12 semanas de reintroducción del gluten en la dieta ${ }^{(23)}$. La adherencia a la dieta libre en gluten durante más de cinco años parece proteger contra el linfoma, cuyo riesgo aumenta hasta 6-10 veces en el momento del diagnóstico ${ }^{(10)}$.

En la actualidad hay dos medicamentos en estudio para el tratamiento de la enfermedad celíaca: acetato de lazarotide y latiglutenasa; sin embargo, hacen falta estudios y guías, por lo que en el momento no se recomiendan sistemáticamente dichos medicamentos (26-27). La dapsona es una opción terapéutica adicional en los pacientes con dermatitis herpetiforme, ya que no reemplaza la dieta libre de gluten, pero ayuda a la mejoría de las manifestaciones en piel en menos tiempo, sin efecto sobre la enteropatía ${ }^{(10)}$. Se utiliza en pacientes con dermatitis herpetiforme durante 6 a 24 meses hasta que la dieta sin gluten sea efectiva. La sulfapiridina y la sulfametoxipiridazina son alternativas descritas válidas ${ }^{(22)}$. Adicionalmente, se recomienda la vacunación contra el neumococo por asociación entre la enfermedad celíaca y la neumonía adquirida en comunidad (28).

En cuanto al pronóstico, la tasa de mortalidad del paciente celíaco está incrementada con relación a la población general debido a la mayor frecuencia de linfoma T intestinal, adenocarcinoma de intestino delgado y carcinomas del tercio superior del esófago. Sin embargo, el pronóstico de los pacientes que se adhieren a la dieta libre de gluten es favorable y la tasa de supervivencia a 10-15 años en estos pacientes no difiere a la de la población general $^{(11)}$.

\section{Conclusiones}

La incidencia de enfermedad celíaca viene en aumento, disminuyendo la calidad de vida de los pacientes que la padecen por su sintomatología y complicaciones, aumentando la mortalidad en relación con la población general. Ya que su presentación clásica es poco frecuente se hace necesario conocer las manifestaciones clínicas no clásicas, entre ellas la dermatitis 
herpetiforme reconocida como el único trastorno dermatológico específico de la enfermedad celíaca. Lo anterior ayudará a un diagnóstico y tratamiento temprano y oportuno de la enfermedad celíaca.

\section{Conflictos de interés}

Ninguno.

\section{Bibliografía}

1. Rubin JE, Crowe SE. Celiac Disease. Ann Intern Med. 2020;172(1): ITC1-16. DOI: 10.7326/AITC202001070

2. Bureš J. History of celiac disease. Vnitr Lek. 2018;64(6):600-1. URL: https://pubmed.ncbi.nlm.nih.gov/30223657/

3. $\mathrm{Yu} X \mathrm{XB}$, Uhde $\mathrm{M}$, Green $\mathrm{PH}$, Alaedini $\mathrm{A}$. Autoantibodies in the Extraintestinal Manifestations of Celiac Disease. Nutrients. 2018;10(8): E1123. DOI: $10.3390 /$ nu10081123

4. Parra-Medina R, Molano-Gonzalez N, Rojas-Villarraga A, Agmon-Levin N, Arango M-T, Shoenfeld $Y$, et al. Prevalence of Celiac Disease in Latin America: A Systematic Review and Meta-Regression. PLOS ONE. 2015;10(5): e0124040. DOI: 10.1371/journal.pone. 0124040

5. Therrien A, Kelly CP, Silvester JA. Celiac Disease: Extraintestinal Manifestations and Associated Conditions. J Clin Gastroenterol. 2020;54(1): 8-21. DOI: 10.1097/MCG.0000000000001267

6. Abenavoli L, Dastoli S, Bennardo L, Boccuto L, Passante M, Silvestri M, et al. The Skin in Celiac Disease Patients: The Other Side of the Coin. Medicina (Mex). 2019;55(9): 578. DOI: $10.3390 /$ medicina55090578

7. Leffler DA, Green PHR, Fasano A. Extraintestinal manifestations of coeliac disease. Nat Rev Gastroenterol Hepatol. 2015;12(10): 561-71. DOI: 10.1038/nrgastro.2015.131

8. Lebwohl B, Sanders DS, Green PHR. Coeliac disease. Lancet Lond Engl. 2018;391(10115): 70-81. 
9. Antiga E, Maglie R, Quintarelli L, Verdelli A, Bonciani D, Bonciolini V, et al. Dermatitis Herpetiformis: Novel Perspectives. Front Immunol. 2019;10: 1290. DOI: 10.3389/fimmu.2019.01290

10. Salmi TT. Dermatitis herpetiformis. Clin Exp Dermatol. 2019;44(7): 728-31. DOI: 10.1111/ced.13992

11. Sanjinés DL. Dermatitis herpetiforme como carta de presentación de la enfermedad celíaca. Rev Urug Med Interna. 2016;7. URL: http://www.scielo.edu.uy/pdf/rumi/v1n1/v1n1a03.pdf

12. J. FM, P. RQ. Enfermedad celiaca: Revisión. Rev Médica Clínica Las Condes. 2015;26(5): 613-27.

13. Rojas-Vargas C. Enfermedad celíaca: una enfermedad autoinmune. Rev Médica Sinerg. 2021;6: 11. DOI: 10.1016/j.rmclc.2015.09.007

14. Jericho H, Sansotta N, Guandalini S. Extraintestinal Manifestations of Celiac Disease: Effectiveness of the Gluten-Free Diet. J Pediatr Gastroenterol Nutr. 2017;65(1):75-9. DOI: 10.1097/MPG.0000000000001420

15. Muddasani S, Rusk AM, Baquerizo Nole KL. Gluten and skin disease beyond dermatitis herpetiformis: a review. Int J Dermatol. 2021;60(3):281-8. DOI: 10.1111/ijd.15098

16. Salmi TT, Hervonen K, Kautiainen H, Collin P, Reunala T. Prevalence and incidence of dermatitis herpetiformis: a 40-year prospective study from Finland. $\mathrm{Br} J$ Dermatol. 2011;165(2): 354-9. DOI: 10.1111/j.1365-2133.2011.10385.x

17. Mansikka E, Hervonen K, Kaukinen K, Collin P, Huhtala H, Reunala T, et al. Prognosis of Dermatitis Herpetiformis Patients with and without Villous Atrophy at Diagnosis. Nutrients. 2018;10(5): E641. DOI: 10.3390/nu10050641

18. Lindfors K, Ciacci C, Kurppa K, Lundin KEA, Makharia GK, Mearin ML, et al. Coeliac disease. Nat Rev Dis Primer. 2019;5(1): 3.

19. Leffler DA, Schuppan D. Update on serologic testing in celiac disease. Am J Gastroenterol. 2010;105(12):2520-4. 
20. Pallav K, Kabbani T, Tariq S, Vanga R, Kelly CP, Leffler DA. Clinical Utility of Celiac DiseaseAssociated HLA Testing. Dig Dis Sci. 2014;59(9): 2199-206. DOI: 10.1038/s41572-0180054-z

21. Ludvigsson JF, Bai JC, Biagi F, Card TR, Ciacci C, Ciclitira PJ, et al. Diagnosis and management of adult coeliac disease: guidelines from the British Society of Gastroenterology. Gut. 2014;63(8): 1210-28. DOI: 10.1136/gutjnl-2013-306578

22. Downey L, Houten R, Murch S, Longson D, Guideline Development Group. Recognition, assessment, and management of coeliac disease: summary of updated NICE guidance. BMJ. 2015;351:h4513.

23. Antiga $E$, Caproni M. The diagnosis and treatment of dermatitis herpetiformis. Clin Cosmet Investig Dermatol. 2015;8:257-65. DOI: 10.2147/CCID.S69127

24. Rodrigo L, Beteta-Gorriti V, Alvarez N, Gómez de Castro C, de Dios A, Palacios L, et al. Cutaneous and Mucosal Manifestations Associated with Celiac Disease. Nutrients. 2018;10(7):E800. DOI: 10.3390/nu10070800

25. Baptista A, Madanelo S, Morais P. A pruritic vesicular rash. Aust Fam Physician. 2017;46(1):45-7.

26. Syage JA, Green PHR, Khosla C, Adelman DC, Sealey-Voyksner JA, Murray JA. Latiglutenase Treatment for Celiac Disease: Symptom and Quality of Life Improvement for Seropositive Patients on a Gluten-Free Diet. GastroHep. 2019;1(6):293-301. DOI: 10.1002/ygh2.371

27. Ja M, Cp K, Phr G, A M, Tt W, M M, et al. No Difference Between Latiglutenase and Placebo in Reducing Villous Atrophy or Improving Symptoms in Patients With Symptomatic Celiac Disease. Gastroenterology. 2021];152(4). URL:https://pubmed.ncbi.nlm.nih.gov/27864127/

28. Zingone F, Abdul Sultan A, Crooks CJ, Tata LJ, Ciacci C, West J. The risk of communityacquired pneumonia among 9803 patients with coeliac disease compared to the general population: a cohort study. Aliment Pharmacol Ther. 2016;44(1):57-67. DOI: 10.1111/apt.13652 